\title{
Radioisotope production at the IFMIF-DONES facility
}

\author{
Javier Praena ${ }^{1, *}$, Francisco Garcia-Infantes ${ }^{1}$, Rafael Rivera ${ }^{1}$, Laura Fernandez-Maza ${ }^{2}$, Fernando Arias de Saavedra $^{1}$, and \\ Ignacio Porras ${ }^{1}$ \\ ${ }^{1}$ Universidad de Granada, Granada, Spain \\ ${ }^{2}$ Hospital Virgen de la Arrixaca, Murcia, Spain
}

\begin{abstract}
The International Fusion Materials Irradiation Facility - Demo Oriented NEutron Source (IFMIFDONES) is a single-sited novel Research Infrastructure for testing, validation and qualification of the materials to be used in a fusion reactor. Recently, IFMIF-DONES has been declared of interest by ESFRI (European Strategy Forum on Research Infrastructures) and its European host city would be Granada (Spain). In spite the first and most important application of IFMIF-DONES related to fusion technology, the unprecedented neutron flux available could be exploited without modifying the routine operation of IFMIF-DONES. Thus, it is already planned an experimental hall for a complementary program with neutrons. Also, a complementary program on the use of the deuteron beam could help IFMIF-DONES to be more sustainable. In the present work, we study radioisotope production with deuterons of ${ }^{177} \mathrm{Lu}$. The results show the viability of IFMIF-DONES for such production in terms of the needs of a territory of small-medium size. Also the study suggests that new nuclear data at higher deuteron energies are mandatory for an accurate study in this field.
\end{abstract}

\section{Introduction}

IFMIF will be an installation for irradiation tests that will simulate the conditions inside a fusion reactor in terms of neutron fluence and neutron energy [1]. In Europe, during the last decades the so-called European Fusion Program has been developed, coordinated between the different European countries and Euratom. In order to reproduce the same hard radiation conditions inside a fusion reactor, it is necessary to build a specific neutron source based on the nuclear reaction that occurs between deuterium and lithium nuclei. The need to build a first fusion reactor, 'early DEMO', has imposed some urgency in the construction of a neutron irradiation plant. It was decided, in the European framework, the design and construction of a plant that was able to generated that level of damage. At the proposal of the National Fusion Laboratory it was decided that it will be DONES (DEMO-Oriented Neutron Source), which basically consists of a simplification of IFMIF. The IFMIF project will consist of two accelerators, however, DONES will consist of a single accelerator in an attempt to achieve this urgency.

Thanks to great efforts many good news have arrived in the last years. For instance, Granada is the european city host the construction of the IFMIF-DONES, and the facility has been recently selected as a key infrastructure in energy by the European Strategy Forum on Research Infrastructures (ESFRI) [2].

The main technological challenges of the installation are the accelerator and the lithium target. The planned accelerator to be included in IFMIF-DONES will accelerate

*e-mail: jpraena@ugr.es with a current of $125 \mathrm{~mA}$ deuterons up to $40 \mathrm{MeV}$, which means that it must handle $5 \mathrm{MW}$ power. The deuteron beams will strike a liquid lithium target that needs to circulate at high speed, $15 \mathrm{~m} / \mathrm{s}$, to extract the high incident power. IFMIF-DONES will reach a neutron flux of $10^{18}$ $\mathrm{m}^{-2} \mathrm{~s}^{-1}$ with a broad peak at $14 \mathrm{MeV}$. In the Figure 1 we can see a scheme of IFMIF-DONES [3].

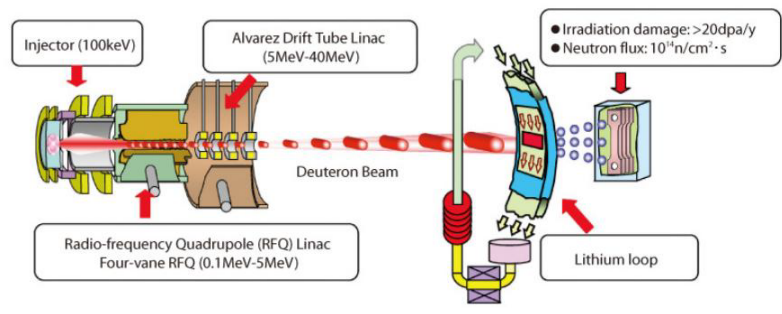

Figure 1. General scheme of operation of IFMIF-DONES.

The materials most exposed in the reactors will be irradiated in the high flux module and in the other two test cells less exposed materials will be irradiated; but the question now is about the possible applications of IFMIFDONES, beyond the study of materials. The White Paper included a preliminary report on a complementary scientific program at IFMIF-DONES [4]. The main part of the report is the compilation of scientific cases for a final Complementary Program ordered by scientific domain: applications of medical interest, nuclear physics and radioactive ion beam installations, basic physics studies and industrial application of neutrons. However, there was not studies regarding the production of radioisotopes with deuterons. In 
this article, we perform a preliminary study on the possible use of IFMIF-DONES for producing medical radioisotopes. We have taken advantage of our theoretical study on the production of radioisotopes with a deuteron accelerator of low energy (3 MeV) and high current (10 mA) [5].

Specifically, for the present work, the study of ${ }^{177} \mathrm{Lu}$ will be of interest to us. This radioisotope has a half-life equal to $6.6465 \mathrm{~d}$ [6], that is, it can be produced and transported without drastically reducing its activity. IFMIFDONES offers the possibility of generating this radioisotope through two production routes, see Figure 2. The first reaction is the direct route (green) and the second is the indirect route (grey).

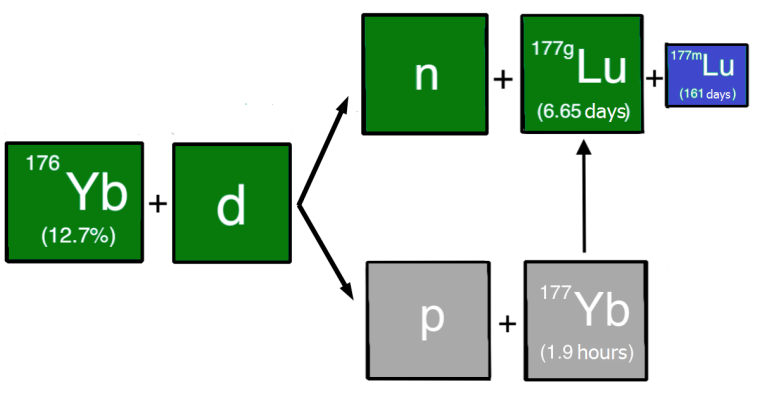

Figure 2. ${ }^{177} \mathrm{Lu}$ production scheme for deuteron-induced reactions on ${ }^{176} \mathrm{Yb}$. The green color is the direct route and the grey color is the indirect route. The blue color highlights the small production of the metastable state of ${ }^{177} \mathrm{Lu}$.

According to the tabulated data of the IAEA [7], the average emission energies of electrons are in the range from 40 to $150 \mathrm{keV}$. This range makes the radioisotope suitable for therapeutic use. On the other hand, during the disintegration the gamma radiation causes it to be detected with imaging techniques. For these reasons, ${ }^{177} \mathrm{Lu}$ is used at present for theranostic (therapy and diagnosis) [8]. Among others, ${ }^{177} \mathrm{Lu}$ is widely used as a radiopharmaceutical to treat gastroenteropancreatic neuroendocrine tumors [9][10][11]. Currently, the ${ }^{177} \mathrm{Lu}$ is only produced in nuclear reactors with two possible reactions [12]. The first is the neutron capture on ${ }^{176} \mathrm{Lu},{ }^{176} \mathrm{Lu}(\mathrm{n}, \gamma){ }^{177(m+g)} \mathrm{Lu}$ with enriched samples, where the products are the same as the direct route with deuterons. The second is the neutron capture on ${ }^{176} \mathrm{Yb}(\mathrm{n}, \gamma){ }^{177} \mathrm{Yb}$ with enriched samples, where the products are the same as the indirect route with deuterons, thus, ${ }^{177} \mathrm{Yb}$ decays to ${ }^{177 g} \mathrm{Lu}$ with no production of the undesirable long-live metastable state. Lutetium is excreted through the urine which must be treated as a nuclear waste for a long time when the metastable state is produced [8]. In case of deuteron production at IFMIFDONES the metastable state would be negligible produced in comparison with nuclear reactors.

In this work, as a first attemp to design a realistic setup for estimating the radioisotope production with deuterons at IFMIF-DONES, it has been considered a simple model for the calculation of the temperature of the target. The target consists of an ytterbium sample deposited onto a copper backing cooled by flowing water. The optimal thick- ness of the sample will be studied with the boundary condition that the temperature must be below the melting point of ytterbium and copper. The current of the deuteron beam will be $1 / 100$ the maximum current for designing issues. It will be shown that in order to continue more accurate studies in the radioisotope production, it is mandatory to new data above $20 \mathrm{MeV}$ on the reaction ${ }^{176} \mathrm{Yb}+\mathrm{d}$.

\section{Materials and method}

The accelerator of IFMIF-DONES open two production routes for the Lu, which should be studied. In order to not melt the target the optimal thickness of $\mathrm{Yb}$ will be calculated as well as the dissipated power. Then, the lutetium production rate and the activity of the lutetium will be studied.

The production of lutetium, through the direct route, is obtained from the differential equation:

$$
\frac{d N_{177_{L u}}}{d t}=R_{177_{L u}}-N_{177_{L u}} \lambda_{177_{L u}}
$$

where $N_{177_{L u}}$ is the number of nuclei produced, $R_{177_{L u}}$ is the rate of production and $\lambda_{177_{L u}}$ is the decay constant. The production rate is given by:

$$
R_{177_{L u}}=\frac{I}{q} \int_{E_{f}}^{E_{i}} \frac{\sigma_{d i r}(E)}{S_{Y b}^{d}(E)} d E
$$

Therefore, the number of nucleus produced remains:

$$
N_{177_{L u}}(t)=\frac{I\left(1-\exp ^{-\lambda_{177_{L u}}}\right)}{q \lambda_{177_{L u}}} \int_{E_{f}}^{E_{i}} \frac{\sigma_{d i r}(E)}{S_{Y b}^{d}(E)} d E
$$

For the indirect route, the differential equation must consider the time of disintegration of the ytterbium. In this way, the differential equation to solve is:

$$
\frac{d N_{177_{L u}}}{d t}=R_{177_{Y b}}\left(1-\exp ^{-\lambda_{177_{L u}}}\right)-N_{177_{L u}} \lambda_{177_{L u}}
$$

The difference with the direct route production rate is also in the cross-section. Therefore, the number of nucleus produced remains:

$$
\begin{gathered}
N_{177_{L u}}(t)=\frac{I}{q} \int_{E_{f}}^{E_{i}} \frac{\sigma_{d i r}(E)}{S_{Y b}^{d}(E)} d E\left(\frac{1-\exp ^{-\lambda_{177_{L u} t}}}{\lambda_{177_{L u}}}+\right. \\
\left.\frac{\exp ^{-\lambda_{177_{Y b}} t}-\exp ^{-\lambda_{177_{L u}} t}}{\lambda_{177_{Y b}}-\lambda_{177_{L u}}}+\frac{1-\exp ^{-\lambda_{177_{Y b}} t}}{\lambda_{177_{L u}}-\lambda_{177_{Y b}}}\right)
\end{gathered}
$$

From here we must solve the two integrals that appear in expressions (3) and (5) [13]. Then, we will calculate the activity of lutetium. 


\subsection{Stopping power}

The stopping power is given by the semi-empirical expression developed by Ziegler and Andersen. This expression was developed for protons, and is given by [14]:

$$
S_{Y b}^{p}(E)=\frac{A_{6}}{\beta^{2}}\left[\operatorname{Ln}\left(\frac{A_{7} \beta^{2}}{1-\beta^{2}}\right)-\beta^{2}-\sum_{i=0}^{4} A_{i+8} \operatorname{Ln}(E)^{i}\right]
$$

where $\beta=\frac{v}{c}, E$ is the energy of the incident beam and all the values of the different $A$ are particular constants of the ytterbium. Taking into account that the stopping power depends on the energy and speed of the incident particle, the relationship between the expression for the stopping power of protons and deuterons is:

$$
S_{Y b}^{p}(E)=S_{Y b}^{d}\left(\frac{m_{p}}{m_{d}}(E)\right)
$$

where $m_{p} \mathrm{y} m_{d}$ are the mass of the proton and deuteron. The same expressions are used by the SRIM code (Stopping and Range of Ions in Matter) [15]. We will use SRIM for a double check of the energy losses by the deuterons in ytterbium.

\subsection{Cross section}

For the values of the cross-section, the experimental data provided by Hermanne et al. [16] and Manenti et al. [17] have been taken. These data range up to $20 \mathrm{MeV}$, so for higher energies we must rely on theoretical fits of the cross-sections, see Figure 3. For the indirect route the fit is (black line):

$\sigma_{\text {in }}(E)=\left\{\begin{array}{l}\frac{d}{s \sqrt{2 \pi}} e^{\frac{-(E-\omega)^{2}}{2 s^{2}}}, 0<E<11.22 \mathrm{MeV} \\ n \cdot e^{\frac{-E}{k}}, 11.22 \mathrm{MeV}<E<40 \mathrm{MeV}\end{array}\right.$

where $d=(1.66 \pm 0.07) 10^{6} \mathrm{mb} \cdot \mathrm{keV}, s=(2.72 \pm 0.16) 10^{3}$ $\mathrm{keV}, u=(1.16 \pm 0.11) 10^{4} \mathrm{keV}, k=(1.19 \pm 0.08) 10^{4} \mathrm{keV}$ and $n=(620 \pm 50) \mathrm{mb}[16]$.

On the other hand, the setting for the fit of the direct route is (red line):

$$
\sigma_{i n}(E)=a+b x
$$

where $a=(-54 \pm 5) \mathrm{mb}$ y $b=(4.4 \pm 0.3) 10^{-3} \mathrm{mb} / \mathrm{keV}$ [17]. Figure 3 shows the fits performed up to $40 \mathrm{MeV}$ for both cross-sections based on the method of our work [5].

\subsection{Target working principle and thermal analysis}

When a beam of energetic charged particles strikes a target a part of the energy is transferred due to the stopping of charged particles. That transfer increases the temperature of the target with the possibility to melt it. To avoid this, a cooling system should be used. In this work, the target structure is composed by 3 sections. The first one is the ytterbium foil, which is attached to the second section,

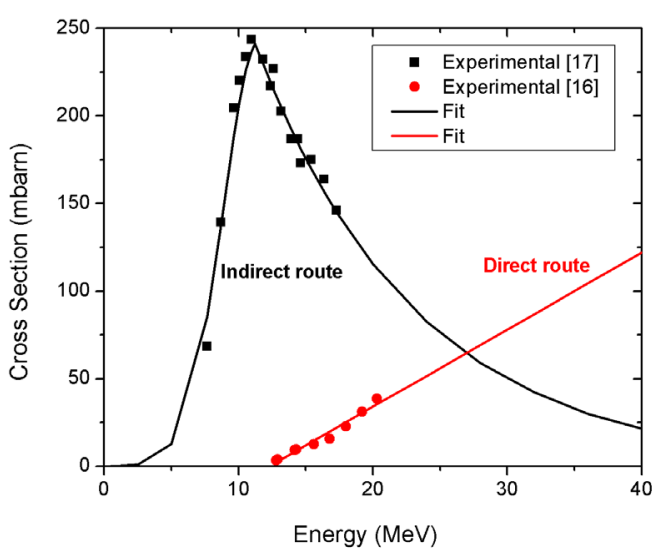

Figure 3. Cross section (mbarn) of the reaction $d+{ }^{176} \mathrm{Yb}$. Square black points correspond to experimental values of the indirect route ${ }^{176} \mathrm{Yb}(\mathrm{d}, \mathrm{p}){ }^{177} \mathrm{Yb} \rightarrow{ }^{177} \mathrm{Lu}$, Manenti et al. [17]. Circle red points corresponds to experimental values of the direct route ${ }^{1776} \mathrm{Yb}(\mathrm{d}, \mathrm{n})^{177(m+g)} \mathrm{Lu}$, Hermanne et al. [16] The lines correspond to the used fit following Arias de Saavedra et al. [5].

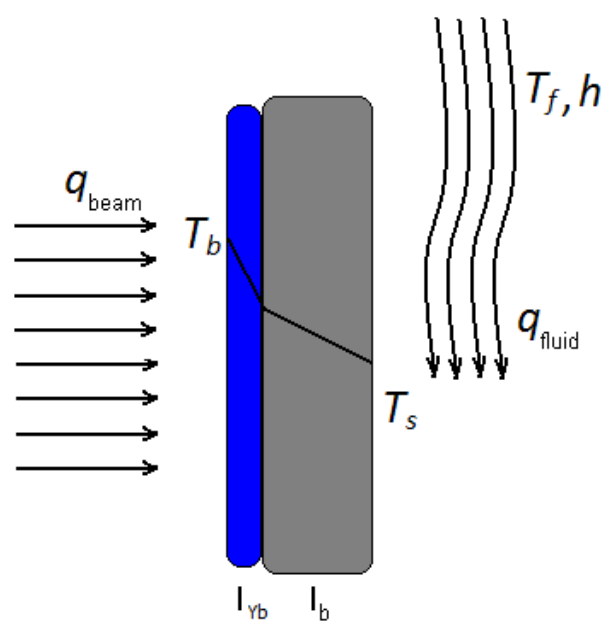

Figure 4. Complete scheme of the considered target composed by ytterbium layer (blue), copper backing (grey) and circulating water (fluid).

the backing. The second section is then in touch or immersed in the third one, the cooling fluid. Figure 4 show schematically the concept of the target.

The thicknesses of the foil and the backing are key parameters because determine the power sustained in each section. This power must be dissipated keeping the temperatures well below the melting points of $\mathrm{Yb}$ and $\mathrm{Cu}$. The dissipation is due to conduction, along the target, and to convection, due to the fluid in contact with the backing. Assuming stationary conditions, the heat dissipated by the fluid is:

$$
q_{\text {fluid }}=h\left(T_{s}-T_{f}\right)
$$


where $h$ is the convection loss coefficient. The convection loss coefficient is related with the Nusselt number by means of the thermal conductivity coefficient and the dimensions of the considered target. Thus, the convection loss coefficient can be calculated as [18]:

$$
h=0.03221 \cdot \frac{k_{f}}{r}\left(\frac{\rho v r}{\mu}\right)^{0.8}\left(\frac{c_{p} \mu}{k_{f}}\right)^{0.43}
$$

where $k_{f}$ is the coefficient of thermal conductivity of the fluid, $r$ is the radius of the target $(1 \mathrm{~cm})$, we have considered the same radius for the deuteron beam, $\rho$ is the density of the water, $\mu$ is the dynamic viscosity of the fluid and $c_{p}$ is the specific heat at constant fluid pressure. On the other hand, the incident heat flow is:

$$
q_{\text {beam }}=\frac{k}{l}\left(T_{Y b}-T_{s}\right)
$$

where $k$ is the coefficient of thermal conductivity of the target-support system and $l$ is the thickness. Therefore, the expression of the incident heat is:

$$
q_{\text {beam }}=\frac{k_{Y b} k_{b}}{k_{Y b} r \sqrt{\frac{0.5 \rho v^{2}+101325}{\sigma_{e}}}+k_{b} l_{Y b}}\left(T_{Y b}-T_{s}\right)
$$

considering $l<<r$ and the conservation of energy, $q_{\text {beam }}=q_{\text {fluid }}=q$, we have the following expression:

$$
q=h_{t}\left(T_{Y b}-T_{f}\right)
$$

where $h_{t}$ is:

$$
\begin{aligned}
h_{t}= & \left(\frac{k_{Y b} 2 r \sqrt{\frac{0.5 \rho v^{2}+101325}{\sigma_{e}}}+k_{b} l_{Y b}}{k_{Y b} k_{b}}+\right. \\
& \left.+27.03 \cdot \frac{2 r}{k_{f}}\left(\frac{\mu}{\rho v r}\right)^{0.8}\left(\frac{k_{f}}{c_{p} \mu}\right)^{0.43}\right)^{-1}
\end{aligned}
$$

\subsection{Activity and specific activity}

The activity of the lutetium sample is given by the expression:

$$
A(t)=\lambda_{177_{L u}} N_{177_{L u}}(T)
$$

where $N_{177_{L u}}(t)$ is the number of generated nucleus of ${ }^{177} \mathrm{Lu}$, which is obtained by adding the result of the expressions 3 and 5. On the other hand, the produced mass of lutetium is given by the expression:

$$
m_{177_{L u}}=N_{177_{L u}} \cdot \frac{176.94 g}{6.022 \cdot 10^{23}}
$$

Finally, having the activity and mass of lutetium after irradiation, we can calculate the specific activity.

\section{Results}

Once we have analyzed all the variables, we can start to make the corresponding calculations. First, we calculate the energy loss by deuterons in ytterbium. From here, we can calculate the temperature reached by the target, which will be the boundary condition. In the following calculations we have considered a copper backing of $0.7 \mathrm{~mm}$ in thickness. The deuterons are stopped in the water or in a second target, which could produce another radioisotope or also ${ }^{177} \mathrm{Lu}$. This option will be developed in forthcoming studies. The cooling water circulates at $5 \mathrm{~m} / \mathrm{s}$ with a temperature of $20^{\circ} \mathrm{C}$. Table 1 shows the average energy lost in $\mathrm{Yb}$, the temperature of the $\mathrm{Yb}$ foil and temperature of the $\mathrm{Cu}$ backing for $40 \mathrm{MeV}$ deuterons at $125 \mathrm{~mA}$ for different ytterbium thicknesses $\left(l_{Y b}\right)$.

\begin{tabular}{cccc}
\hline$\Delta E \mathrm{MeV}$ & $l_{Y b}(\mathrm{~mm})$ & $\mathrm{T}_{Y b}(\mathrm{~K})$ & $\mathrm{T}_{C u}(\mathrm{~K})$ \\
\hline 9.020 & 1.000 & $622 \cdot 10^{3}$ & $518 \cdot 10^{3}$ \\
4.305 & 0.500 & $273 \cdot 10^{3}$ & $247 \cdot 10^{3}$ \\
0.833 & 0.100 & $49 \cdot 10^{3}$ & $47 \cdot 10^{3}$ \\
0.414 & 0.050 & $24 \cdot 10^{3}$ & $23.8 \cdot 10^{3}$ \\
0.083 & 0.010 & $15 \cdot 10^{3}$ & $14.9 \cdot 10^{3}$ \\
\hline
\end{tabular}

Table 1. Average energy lost in ytterbium of $40 \mathrm{MeV}$ deuterons at $I=125 \mathrm{~mA}$, the currently expected characteristics of IFMIF-DONES ion beam. Different thicknesses of $\mathrm{Yb}$ are considered. Copper thickness is fixed to $0.7 \mathrm{~mm}$. Water is kept at $20^{\circ} \mathrm{C}$ and $5 \mathrm{~m} / \mathrm{s}$.

The melting temperature of ytterbium is $\mathrm{T}_{Y b}=1096$ $\mathrm{K}$ and for copper is $\mathrm{T}_{C u}=1358 \mathrm{~K}$. Then, the production of lutetium for an intensity of $I=125 \mathrm{~mA}$ is unfeasible. Therefore, the limitations in the deuteron current imposed by the temperatures are remarkable. For a future complementary program on the production of radioisotopes with deuterons, the reduction of the intensity of the incident beam should be considered. Thus, considering the same model, for an intensity $I=1.25 \mathrm{~mA}$, neither the ytterbium foil nor the copper backing are melt, if the $\mathrm{Yb}$ thickness is less than $l_{Y b}=200 \mu \mathrm{m}$.

One of the aims of the present work is to compare the analytical model for radioisotope production with deuterons to the current production at nuclear reactors by means of neutron capture reactions. In case of nuclear reactors, the chemical form of the $\mathrm{Yb}$ sample is $\mathrm{Yb}_{2} \mathrm{O}_{3}$ with a maximum possible enrichment of $97 \%$ [19]. In the following, we calculate the production rates, mass and activity of $97 \%$ enriched $\mathrm{Yb}_{2} \mathrm{O}_{3}$ and ytterbium samples. The melting point of $\mathrm{Yb}_{2} \mathrm{O}_{3}$ is higher than natural ytterbium, while the thermal conductivity is lower [20]. Nevertheless, our calculations have kept as boundary condition the temperature of the foils, either oxide or natural, well below their melting points. In the following results, we have considered $40 \mathrm{MeV}$ deuteron energy, $I=1.25 \mathrm{~mA}$ deuteron current, $\mathrm{Yb}$ thickness of $150 \mu \mathrm{m}, \mathrm{Cu}$ backing thickness of $0.7 \mathrm{~mm}$, and temperature and velocity of water $20^{\circ} \mathrm{C}$ and $5 \mathrm{~m} / \mathrm{s}$, respectively. Table 2 shows the results of the production rate of ${ }^{177} \mathrm{Lu}$ with $\mathrm{Yb}$ and $\mathrm{Yb}_{2} \mathrm{O}_{3}$ samples and it 
summarizes the main parameters used in our model (first and second columns).

\begin{tabular}{lcc}
\hline$R_{L u}(\mathrm{mg} / \mathrm{s})$ & ${ }^{196} \mathrm{Yb}$ & ${ }^{196} \mathrm{Yb}_{2} \mathrm{O}_{3}$ \\
\hline Direct route & $1.76 \cdot 10^{-7}$ & $3.08 \cdot 10^{-7}$ \\
Indirect route & $3.35 \cdot 10^{-8}$ & $6.03 \cdot 10^{-8}$ \\
\hline
\end{tabular}

Table 2. Values obtained for the production rate of ${ }^{177} \mathrm{Lu}$ in milligram per second, $R_{L u}(\mathrm{mg} / \mathrm{s})$, with a $\mathrm{Yb}$ sample and a

$\mathrm{Yb}_{2} \mathrm{O}_{3}$ sample of $150 \mu \mathrm{m}$ thickness. Parameters: $40 \mathrm{MeV}$ deuterons, $I=1.25 \mathrm{~mA}$, enrichment $97 \%$ in ${ }^{196} \mathrm{Yb}$, temperature of water $20^{\circ} \mathrm{C}$, velocity of water $5 \mathrm{~m} / \mathrm{s}$, thickness of $\mathrm{Cu} 0.7 \mathrm{~mm}$.

The higher production rate obtained for the ${ }^{196} \mathrm{Yb}_{2} \mathrm{O}_{3}$ is due to its higher density than the natural ytterbium. With the same parameters, we have calculated the mass produced by lutetium after irradiation, in Figure 5 the results are shown as a function of time, up to 24 hours of irradiation. Figure 6 shows the results obtained for the generated lutetium activity.

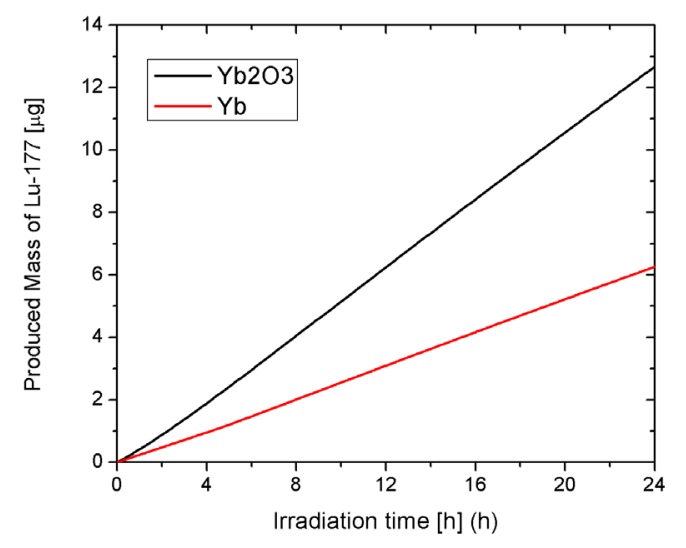

Figure 5. Produced mass of ${ }^{177} \mathrm{Lu}$ for ${ }^{176} \mathrm{Yb}$ foil and ${ }^{196} \mathrm{Yb}_{2} \mathrm{O}_{3}$ foil. Both, direct and indirect routes are included in the calculation. Parameters: $40 \mathrm{MeV}$ deuterons, $I=1.25 \mathrm{~mA}, 97 \%$ enrichment of ${ }^{196} \mathrm{Yb}$ in the samples, temperature of water $20^{\circ} \mathrm{C}$, velocity of water $5 \mathrm{~m} / \mathrm{s}$, thickness of $\mathrm{Cu} 0.7 \mathrm{~mm}$.

\section{Discussion}

The specific activity obtained after $24 \mathrm{~h}$ at IFMIF-DONES is $0.119 \mathrm{GBq} / \mathrm{mg}$ for the ${ }^{196} \mathrm{Yb}_{2} \mathrm{O}_{3}$ sample, and 0.076 $\mathrm{GBq} / \mathrm{mg}$ for the ${ }^{196} \mathrm{Yb}$ sample. We can compare these values to the specific activity produced in nuclear reactors with ${ }^{196} \mathrm{Yb}_{2} \mathrm{O}_{3}$ sample after an irradiation of $72 \mathrm{~h}$, which is $2.96 \mathrm{TBq} / \mathrm{mg}$ [19]. Although the specific activity is lower, the production at IFMIF-DONES would have a considerable impact in a regional health system as Granada (Spain). Conventionally a patient needs four dosis during a treatment. Each dose has a price of 14 kEuros per dose and an activity of $7.4 \mathrm{GBq}$ per dose [21]. Therefore, in case of $24 \mathrm{~h}$ of irradiation at IFMIF-DONES of a ${ }^{196} \mathrm{Yb}_{2} \mathrm{O}_{3}$ sample as considered in the present work, the produced

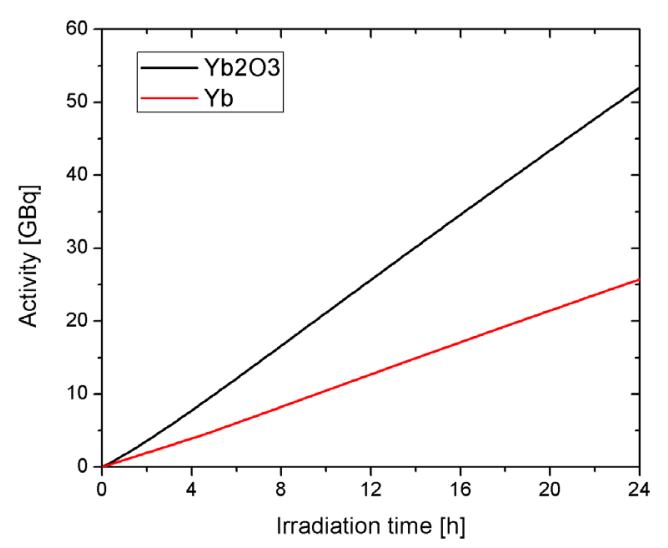

Figure 6. Activity generated by ${ }^{177} \mathrm{Lu}$. Parameters: $40 \mathrm{MeV}$ deuterons, $I=1.25 \mathrm{~mA}, 97 \%$ enrichment of ${ }^{196} \mathrm{Yb}$ in the samples, temperature of water $20^{\circ} \mathrm{C}$, velocity of water $5 \mathrm{~m} / \mathrm{s}$, thickness of $\mathrm{Cu} 0.7 \mathrm{~mm}$.

${ }^{177} \mathrm{Lu}$ could save 112 kEuros. It should be stressed that we are only providing preliminary calculations with the aim to motivate more realistic studies.

With the same idea, we have studied with the same model other radioisotopes of interest in diagnosis and therapy that could be produced at IFMIF-DONES facility. The thicknesses of each reaction was selected to keep the temperature well below the melting point of the corresponding element and the $\mathrm{Cu}$ backing. The rest of parameters are the same of the present study on ${ }^{177} \mathrm{Lu}$. The idea behind is the same, to provide a preliminary study of their production for motivating a possible discussion on a radioisotope complementary program. Table 3 shows a resume of the results considering the same parameters as Table 2.

\begin{tabular}{lcc}
\hline Reaction & Activity $(\mathrm{GBq})$ & Mass $(\mathrm{mg})$ \\
\hline${ }^{64} \mathrm{Ni}(\mathrm{d}, 2 \mathrm{n}){ }^{64} \mathrm{Cu}$ & $2.21 \cdot 10^{6}$ & 15.52 \\
${ }^{n a t} \mathrm{Zn}(\mathrm{d}, 2 \mathrm{n}){ }^{64} \mathrm{Cu}$ & $6.05 \cdot 10^{-3}$ & $4.23 \cdot 10^{-3}$ \\
${ }^{103} \mathrm{Rh}(\mathrm{d}, 2 \mathrm{n}){ }^{103} \mathrm{Pd}$ & $2.51 \cdot 10^{1}$ & $6.26 \cdot 10^{-3}$ \\
${ }^{124} \mathrm{Te}(\mathrm{d}, 2 \mathrm{n}){ }^{124} \mathrm{I}$ & $8.94 \cdot 10^{5}$ & 96.28 \\
${ }^{169} \mathrm{Tm}(\mathrm{d}, 2 \mathrm{n}){ }^{169} \mathrm{Yb}$ & $2.52 \cdot 10^{5}$ & 282.62 \\
${ }^{192} \mathrm{Os}(\mathrm{d}, 2 \mathrm{n}){ }^{192(m+g)} \mathrm{Ir}$ & $2.57 \cdot 10^{1}$ & $6.26 \cdot 10^{-3}$ \\
\hline
\end{tabular}

Table 3. Activity (GBq) and mass (mg) of the radioisotope produced in each reaction. The considered enrichment is $100 \%$ except for $\mathrm{Zn}, \mathrm{Tm}$ and $\mathrm{Rh}$, which is natural abundance.

Finally, it is important to mention in the framework of the 2019 International Conference On Nuclear Data, that there are a need of experimental data for the lutetium production and for many reactions considered in Table 3. Briefly, there is no data above $25 \mathrm{MeV}$ for the ${ }^{64} \mathrm{Ni}(\mathrm{d}, 2 \mathrm{n}){ }^{64} \mathrm{Cu},{ }^{\text {nat }} \mathrm{Zn}(\mathrm{d}, 2 \mathrm{n}){ }^{64} \mathrm{Cu}$ and ${ }^{124} \mathrm{Te}(\mathrm{d}, 2 \mathrm{n}){ }^{124} \mathrm{I}$ reactions [22]. 


\section{Conclusions}

We have studied the production of radioisotopes with $40 \mathrm{MeV}$ deuterons at IFMIF-DONES. Specifically, for ${ }^{177} \mathrm{Lu}$ at present used in diagnosis and imaging of cancers, two reactions has been analyzed: the indirect route ${ }^{176} \mathrm{Yb}(\mathrm{d}, \mathrm{p}){ }^{177} \mathrm{Yb} \rightarrow{ }^{177} \mathrm{Lu}$, and the direct route ${ }^{1776} \mathrm{Yb}(\mathrm{d}, \mathrm{n})^{177(m+g)} \mathrm{Lu}$. A cooling system has been studied with an analytical model. In order to avoid the melting of the target, the current should be decreased to $1.25 \mathrm{~mA}$. The produced ${ }^{177} \mathrm{Lu}$ in $24 \mathrm{~h}$ could have a significant impact in the health system at regional level. The nuclear data needs on deuteron-induced reactions for calculating the possible production at IFMIF-DONES have been shown. Our next step will be to perform a study with Solidworks using a real target. Also, the possibility to multiple irradiation with the same deuteron beam will be studied as well as the production of impurities.

Acknowlegments. This work has been carried out within the framework of the EUROfusion Consortium and has received funding from the Euratom research and training programme 2014-2018 and 2019-2020 under grant agreement No 633053. The views and opinions expressed herein do not necessarily reflect those of the European Commission. This work was supported by the Spanish projects FIS2015-69941-C2-1-P (MINECO-FEDER, EU), A-FQM-371-UGR18 (Prog. Operativo FEDER Andalucia 2014-2020), the Spanish Association Against Cancer (AECC) (Grant No. PS16163811PORR), and the sponsors of the University of Granada Chair Neutrons for Medicine: Fundación ACS, Capitán Antonio and La Kuadrilla.

\section{References}

[1] F. Mota et al., Sensitivity of IFMIF-DONES irradiation characteristics to different design parameters Nucl. Fusion 55 (2015).

[2] http://www.roadmap2018.esfri.eu/projects-andlandmarks/browse-the-catalogue/ifmif-dones/

[3] J. Knaster et al., IFMIF, the European-Japanese effors under the Broader Approach agreement towards a $\operatorname{Li}(d, x n)$ neutron source: Current status and future options, Nucl. Materials and Energy 9 (2016) 46-54.

[4] A. Maj et al., White Book on the Complementary Scientific Programme at IFMIF-DONES. www.ifj. edu.pl/publ/reports/2016/

[5] F. Arias de Saavedra, I. Porras and J. Praena, Routes for the production of isotopes for PET with high intensity deuteron accelerators. Nuclear Inst. and Methods in Physics Research, A 887 (2018) 50-53.

[6] S. Pommé et al., Measurement of the ${ }^{177} \mathrm{Lu}$ Half-life, Applied Rad. and Isotopes. 69 (2011) 1267-1273.

[7] https://www-nds.iaea.org/relnsd/vcharthtml/ VChartHTML.html
[8] M. Weineisen, M. Schottelius et al., ${ }^{68} \mathrm{Ga}-$ and ${ }^{177} \mathrm{Lu}-$ Labeled PSMA I\&T: Optimization of a PSMA targeted theranostic concept and first proof of concept human studies, Journal of Nuclear Medicine 56 (2015) 1169-1176.

[9] J. Kwekkeboom, W. de Herder et al., Treatment With the Radio labeled Somatostatin Analog [ ${ }^{177} \mathrm{Lu}-$ DOTA0,Tyr3] Octreotate: Toxicity, Efficacy, and Survival, Journal of Clinical Oncology. 28 (2008) 2124-2130.

[10] J. Kwekkeboom, J. Teunissen et al., Radiolabeled Somatostatin Analog [ ${ }^{177}$ Lu- DOTA0,Tyr3] Octreotate in Patients With Endocrine Gastroenteropancreatic Tumors, J. of Clinical Oncology. 23 (2005) 2754-2762.

[11] J. Strosberg, et al., Phase 3 Trial of ${ }^{177}$ Lu-Dotatate for Midgut Neuroendocrine Tumors, The New England J. of Medicine. 376 (2017) 125-135.

[12] M.R.A Pillai, S. Chakraborty, T. Das,M. Venkatesh, N. Ramamoorthy. Production logistics of ${ }^{177} \mathrm{Lu}$ for radionuclidetherapy. Applied Radiation and Isotopes 59 (2-3), 109-118 (2003).

[13] A. Dash et al., Production of ${ }^{177} L u$ for Targeted Radionuclide Therapy: Available Options, Nuclear Medicine Molecular Imaging 49 (2015) 85-107.

[14] H. H. Andersen, J. F. Ziegler, Stopping Powers and Ranges in All Elements, Pergamon Press, NY, 1985.

[15] http://www.srim.org/

[16] A. Hermanne et al., Deuteron-induced reactions on $Y b$ : Measured cross sections and rationale for production pathways of carrier-free, medically relevant radionuclides, Nuclear Instruments and Methods in Physics Research B. 247 (2006) 223-231.

[17] S. Manenti et al., Excitation function for deuteron induced nuclear reactions on natural ytterbium for production of high specific activity ${ }^{177 g} \mathrm{Lu}$ in no-carrieradded form for metabolic radiotherapy, Applied Radiation and Isotopes. 69 (2011) 37-45.

[18] J. H. Lienhard IV, J. H. Lienhard V. A heat transfer text book. $3^{\text {rd }}$ ed. Cambridge, MA. Phlogiston, Press, c2008.

[19] Ashutosh Dash, Maroor Raghavan Ambikalmajan Pillai, Furn F. Knapp Jr.. Review. Production of ${ }^{177} \mathrm{Lu}$ for Targeted Radionuclide Therapy: Available Options. Nucl. Med. Mol. Imaging (2015) 49:85-107.

[20] Leilei Sun, et al., Phase stability and thermal conductivity of ytterbia and yttria co-dopedzirconia. Progress in Natural Science: Materials International 2013 ; 23(4) :440-445.

[21] Agencia Española de Medicamentos y Productos Sanitarios, Spanish Government, https://cima.aemps.es/cima/publico/home.html

[22] https://www.nndc.bnl.gov/exfor/exfor.htm 